\title{
Prótesis implantables de oído medio
}

\author{
Implantable prothesis of the middle ear
}

Ursula Zelada Bacigalupo¹.

\begin{abstract}
RESUMEN
Un importante número de pacientes con hipoacusia requiere de la adaptación de una prótesis auditiva, sin embargo, por distintos motivos, muchos de ellos no se sienten satisfechos con las prótesis auditivas convencionales. Una alternativa para estos pacientes son las prótesis implantables de oído medio, dispositivos electrónicos que se implantan parcial o totalmente mediante un procedimiento quirúrgico en el oído medio y que resuelven en gran medida las incomodidades que aquejan a los usuarios de un audífono convencional.
\end{abstract}

Palabras claves: Hipoacusia, implantes, oído medio.

\section{SUMMARY}

An important number of patients with hypoacousia require the adaptation of an auditive prothesis, however, for different reasons, many of them are not satisfied with the conventional auditive prothesis. One alternative for these patients are the implantable prothesis in the middle ear; electronic devices that are total or partially implanted through a surgical procedure in the middle ear, which solve in great measure the discomfort suffered by the users of a conventional hearing aid.

Key words: Hypoacousia, implants, middle ear.

\section{INTRODUCCIÓN}

La hipoacusia, la tercera condición crónica más prevalente en el adulto, provoca importantes efectos en la salud físico-mental y calidad de vida de los pacientes afectados. En alrededor de un $20 \%$ de las hipoacusias, se requiere de la adaptación de una prótesis auditiva, sin embargo, un importante número de estos pacientes no la usa o se sienten insatisfechos con ella. Se estima, según la literatura, que un $15 \%$ de los pacientes con hipoacusia moderada-grave no toleran las prótesis auditivas de conducción aérea y alrededor del $64 \%$ se encuentra disconformes con ellas.

Las razones para el no uso de los audífonos convencionales son las siguientes: incomodidad de la adaptación, ganancia funcional limitada, presencia de retroalimentación acústica, distorsión sono-

${ }^{1}$ Médico del Servicio de Otorrinolaringología del Hospital Barros Luco Trudeau 
ra, infecciones recurrentes del conducto auditivo externo y factores cosméticos.

Una alternativa terapéutica para estos pacientes son las prótesis implantables de oído medio.

\section{Prótesis implantables de oído medio}

Las prótesis implantables de oído medio, son dispositivos electrónicos que se implantan total o parcialmente en el oído medio, mediante cirugía, con el fin de provocar vibración en la cadena osicular y así estimular el oído interno.

A actuar aplicando energía mecánica directamente sobre los huesecillos de la cadena osicular, la transmisión de la energía es más eficiente y con menor distorsión: amplían los movimientos de la cadena osicular, dentro de márgenes de seguridad, logrando estimular directamente el oído interno y evitando los fenómenos de distorsión provocados por la estimulación acústica aplicada en el conducto auditivo externo como es en el caso de los audífonos convencionales.

日 rango de frecuencias sobre el que actúan las prótesis de oído medio, dependiendo del modelo, está comprendido entre los $400 \mathrm{~Hz}$ y los 10.000 $\mathrm{Hz}$, si bien, su máxima eficacia es en las frecuencias de la palabra hablada En relación a la ganancia auditiva (diferencia entre la intensidad de entrada y de salida de la señal procesada), las prótesis implantables generan intensidades de salida hasta $115 \mathrm{~dB}$ SPL, lo que permite su uso en el tratamiento de hipoacusias moderadas a graves.

\section{CLASIFICACIÓN}

Una prótesis implantable de oído medio está compuesta por un micrófono, un audioprocesador, una batería, una unidad receptora y un transductor. Existen tres grupos de transductores: piezoeléctricos, electromagnéticos y electromecánicos (Tabla 1).

1) Los transductores piezoeléctricos utilizan las propiedades de ciertos materiales, como las cerámicas, que tienen la capacidad de deformarse cuando se les aplica una corriente eléctrica; esta deformación será el estímulo mecánico para la cadena osicular. Existen dos tipos: los monomorfos, en los que el movimiento es por expansión-contracción del material y los dimorfos, en los que hay dos fragmentos de material con polaridades diferentes, lo que provoca un desplazamiento lateral.

2) Los transductores electromagnéticos utilizan un imán y una bobina generadora de un campo eléctrico. 日 imán se incorpora a la cadena osicular (lo más frecuente), a la membrana timpánica o a la ventana redonda. La bobina al activarse a partir de una señal acústica, genera un campo magnético que provocará el desplazamiento del imán, el cual pondrá en movimiento la cadena osicular y estimulará el oído interno (Figuras 1, 2, 3).

3) Los transductores electromecánicos son una variación de los electromagnéticos, unen la bobinay el imán para tener un mejor control de

Tabla 1. Implantes de oído medio, aprobados o en fase de aprobación por la FDA y CE

\begin{tabular}{|lccc|}
\hline IOM & Gase de transductor & Tipo de implante & FDA/CE \\
\hline Symphonix & Eectromagnético & Parcial & Sí/sí \\
Otologicx & Eectromagnético & Parcial & Pendiente \\
Soundtex & Eectromagnético & Parcial & Sí/pendiente \\
St. Oroix Medical & Piezoeléctrico & Completo & Pendiente \\
Implex & Piezoeléctrico & Completo & Pendiente/sí \\
\hline
\end{tabular}




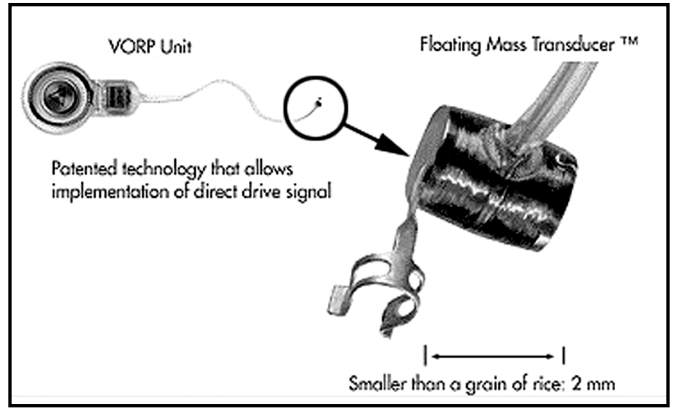

Figura 1.

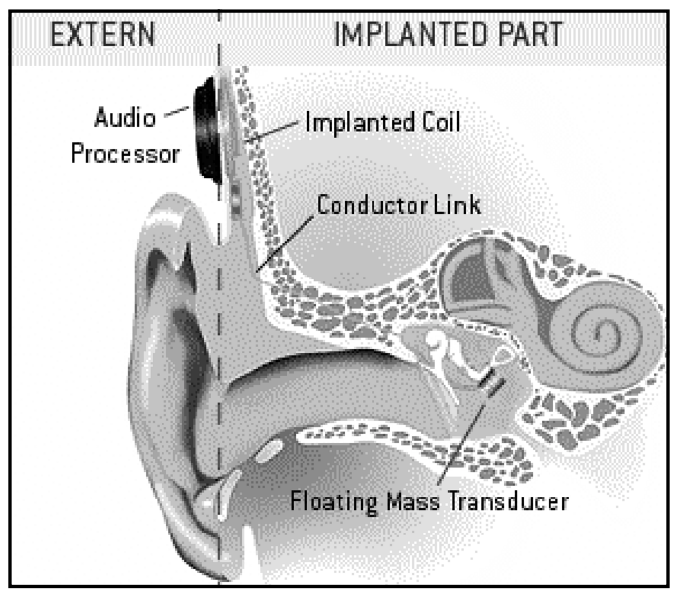

Figura 2.

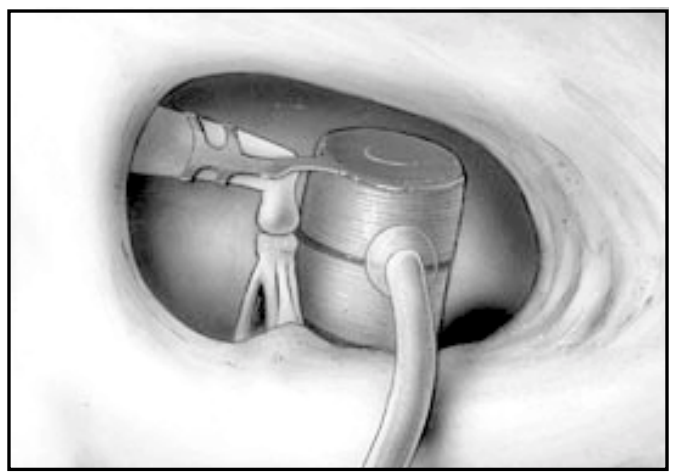

Figura 2 .

las fluctuaciones energéticas y transmiten la vibración a la cadena osicular mediante un sistema mecánico.

\section{INDICACIONES}

Las prótesis auditivas están indicadas en todos aquellos pacientes con hipoacusia neurosensorial moderada a grave, que no se beneficien de una prótesis auditiva de estimulación acústica aérea $\mathrm{Si}$ bien, según el tipo de prótesis se ha definido el tipo de paciente ideal, en general se consideran los siguientes requisitos:

- Ser mayor de 18 años.

- Hipoacusia bilateral neurosensorial, con umbrales de conducción aérea máximos o dentro de los límites establecidos.

- Diferencia aéreo-óseo en las frecuencias de $500,1.000,2.000$ y $4.000 \mathrm{~Hz}$ menor o igual a $10 \mathrm{~dB}$, en al menos dos de estas frecuencias.

- Reconocimiento mínimo del habla de un $50 \%$ o mejor.

- Funcionamiento y anatomía normal del oído medio.

- Sin antecedentes quirúrgicos de oído medio, infecciones crónicas o alteraciones anatómicas de oído interno.

- Que no exista ninguna evidencia de hipoacusia de origen retrococlear.

- Insatisfacción comprobada con pruebas auditivas del uso de prótesis auditiva convencional, al menos durante seis meses en el oído a implantar.

- Expectativas realistas y ausencia de alteraciones vestibulares.

\section{VENTAJAS Y DESVENTAJAS}

Estos dispositivos presentan las siguientes ventajas en relación a los audífonos convencionales: evitan los problemas de retroalimentación o feedback, al mejorar la transmisión de la energía sonora proporcionan una audición más natural, con mejor discriminación en ambiente ruidoso, mantienen el conducto auditivo externo libre, evitando los problemas de oclusión y anulando las molestias o contraindicaciones que plantean las enfermedades de éste y finalmente, son estéticamente más aceptables.

Por el contrario, las prótesis implantables presentan algunos inconvenientes: precisan de un 
procedimiento quirúrgico con anestesia general, existe la posibilidad de daño sobre la cadena osicular, en caso de falla en su funcionamiento puede requerirse de nuevas cirugías, algunos dispositivos limitan la práctica de resonancia nuclear magnética y terapia electroconvulsiva y tienen un costo económico elevado.

\section{BIBLIOGRAFÍA}

1. Smeth L, fletcher AE, Ng ES et al. Reduced hearing, ownership, and use of hearing aids in elderly people in the UK-the MRC Trial of the Assessment and Management of Oder People in the Community: a cross-sectional survey. Lancet 2002; 359(9316): 1466-70.

2. GERSDORF M, GARIN P, LURQUIN P. Implantable hearing aids vs classic hearing aids. Acta Otorhinolaryngol Belg 2002; 56(4): 363.

3. Yuet B, SHAPIRO N, Madlean CH, SHeKElE PG Screening and management of adult hearing loss in primary care: scientific review. JAMA 2003; 289(15): 1976-85.

4. JENKINS HA, NIPARKO JK, SLATTERY WH, N⿴囗十 JG, FREDRICKSON JM. Otologics Middle Ear Transducer Ossicular Stimulator: performance results with varying degrees of sensorineural hearing loss. Acta Atolaryngol 2004; 124(4): 391-4.

5. FEgrsPIL PA, PLINKERT PK. Restoring hearing with active hearing implants. Biomed Tech (Berl) 2004; 49(4): 78-82.

6. UZIE A, Mondain M, Hagen P, DEJEAN F, DOUCET $G$ Rehabilitation for high-frequency sensori- neural hearing impairment in adults with the symphonix vibrant soundbridge: a comparative study. Otol Neurotol 2003; 24(5): 775-83.

7. StgRkers O, BOucarRA D, LABASSI S ET AL. A middle ear implant, the Symphonix Vibrant Soundbridge: retrospective study of the first 125 patients implanted in France. Otol Neurotol 2003; 24(3): 427-36.

8. W由ER PC. Medical and surgical considerations for implantable hearing prosthetic devices. $A m$ J Audiol 2002; 11(2): 134-8.

9. SPINDE JH. Middle ear implantable hearing devices. Am J Audiol 2002; 11(2): 104-13.

10. HOUGH JV, MATTHEWS P, WOOD MW, DYER RKJR. Middle ear electromagnetic semi-implantable hearing device: results of the phase II SOUNDTEC direct system clinical trial. Otol Neurotol 2002; 23(6): 895-903.

11. JAFE IS. Implantable middle ear hearing devices: Food and Drug Administration review process. Otolaryngol Cin North Am 2001; 34(2): 515-7.

12. KASIC JF, FREDRICKSON JM. The Otologics MET ossicular stimulator. Otolaryngol Cin North Am 2001; 34(2): 501-13.

13. ZENNER HP, LEYSIETR H. Total implantation of the Implex TICA hearing amplifier implant for high frequency sensorineural hearing loss: the Tubingen University experience. Otolaryngol Cin North Am 2001; 34(2): 417-46. Review.

14. HOUGH JV, DYER RKJR, MATTHEWS P, WoOD MW. Semi-implantable electromagnetic middle ear hearing device for moderate to severe sensorineural hearing loss. Otolaryngol Cin North Am 2001; 34(2): 401-16.

Dirección: Dra. Ursula Zelada B.

Jorge Washington 260, Depto. 307. Santiago - Chile

Email: ursu_z@yahoo.com 